\title{
On the Absolute Trace of Polynomials Having All Zeros in a Sector
}

\author{
V. Flammang
}

\section{CONTENTS}

\section{Introduction}

2. The Explicit Auxiliary Functions

3. Auxiliary Functions and Integer Transfinite Diameter

4. Construction of the Auxiliary Functions

5. Conclusion

Acknowledgments

References
2000 AMS Subject Classification: Primary 11R04, 11Y40, 12D10

Keywords: Algebraic integer, trace, explicit auxiliary functions, integer transfinite diameter
Let $\alpha$ be an algebraic integer all of whose conjugates lie in a sector $|\arg z| \leq \theta$ with $0 \leq \theta<90^{\circ}$. Using the method of explicit auxiliary functions, we compute the greatest lower bound $v(\theta)$ of the absolute trace of $\alpha$, for $\theta$ belonging to seven subintervals of $\left[0,90^{\circ}\right)$. The polynomials involved in the auxiliary functions are found by Wu's algorithm.

\section{INTRODUCTION}

Let $\alpha$ be a nonzero algebraic integer of degree $d \geq 1$ whose conjugates are $\alpha_{1}=\alpha, \ldots, \alpha_{d}$.

The Mahler measure of $\alpha$ is

$$
M(\alpha)=\prod_{i=1}^{d} \max \left(1,\left|\alpha_{i}\right|\right),
$$

and the absolute Mahler measure of $\alpha$ is $\Omega(\alpha)=M(\alpha)^{1 / d}$. Another well-known representation for the Mahler measure is

$$
h(\alpha)=\frac{1}{d} \log M(\alpha)=\log \Omega(\alpha),
$$

where $h(\alpha)$ is the Weil height of $\alpha$.

The set of algebraic numbers equipped with the Weil height satisfies the Northcott property: there are only finitely many algebraic numbers with bounded degree and bounded height (for more details, see the survey [Bombieri 08]). It is interesting to study the points of this set of small height (or "small points").

The well-known Lehmer's question is the following: Does there exist a positive constant $c>1$ such that $M(\alpha)>c$ for $\alpha$ a nonzero algebraic number not a root of unity? The smallest known value for $M(\alpha)>1$ was found by D. H. Lehmer himself [Lehmer 33] and is $M(\alpha)=1.176280 \ldots$, where the minimal polynomial of $\alpha$ is $P=X^{10}+X^{9}-X^{7}-X^{6}-X^{5}-X^{4}-X^{3}+X+1$.

In 1971, C. J. Smyth [Smyth 71] solved Lehmer's question in the special case of nonreciprocal algebraic numbers. Recall that an algebraic number is reciprocal if 
its minimal polynomial $P$ is a reciprocal polynomial: $P(X)=X^{\operatorname{deg}(P)} P\left(\frac{1}{X}\right)$. He proved that if the algebraic number $\alpha \neq 0,1$ is not reciprocal, then $M(\alpha) \geq \theta_{0}$, where $\theta_{0}=1.324717 \ldots$ is the smallest Pisot number that is the real root of $X^{3}-X-1=0$.

Lower bounds have also been found for special algebraic numbers. We say that an algebraic integer $\alpha$ is totally positive if $\alpha$ and all its conjugates are real positive numbers. A. Schinzel [Schinzel 73] proved that if $\alpha$ is a totally positive algebraic integer, $\alpha \neq 0,1$, then $M(\alpha) \geq\left(\frac{1+\sqrt{5}}{2}\right)^{d}$.

In another direction, F. Amoroso and R. Dvornicich [Amoroso and Dvornicich 00] proved that if $\alpha$ is an algebraic number of degree $d$, not a root of unity, whose splitting field is an abelian extension of $\mathbb{Q}$, then $M(\alpha) \geq 5^{d / 12}$.

Schinzel's result above gives the smallest point of the set of totally positive algebraic integers equipped with the Mahler measure. In 1981, C. J. Smyth [Smyth 81b] gave the three smallest points. The author gave two more points [Flammang 96]. In both cases, heuristic methods were used to find these points.

Then the study of "small points" was extended to the sets $E_{\theta}$ of algebraic integers $\alpha$ all of whose conjugates lie in a sector $S_{\theta}=\{z \in \mathbb{C}:|\arg z| \leq \theta\}$ with $0 \leq \theta<$ $180^{\circ}$. Here we need to define the transfinite diameter of a compact subset $K$ of $\mathbb{C}$ :

$$
t(K)=\liminf _{\substack{n \geq 1 \\ n \rightarrow+\infty}} \inf _{\substack{P \in \mathbb{C}[X] \\ \operatorname{deg}(P)=n}}|P|_{\infty, K}^{1 / n},
$$

where we set $|P|_{\infty, K}=\sup _{z \in K}|P(z)|$ for $P \in \mathbb{C}[X]$.

In this definition, if we replace $P \in \mathbb{C}[X], P$ monic, by $P \in \mathbb{Z}[X]$, we get the definition of the integer transfinite diameter of $K$.

A theorem of M. Langevin [Langevin 86] based on the fact that for $\theta<180^{\circ}$, the transfinite diameter of $S(\theta) \cap\{z \in \mathbb{C}:|z| \leq 1\}$ is less than 1 proves that there exists a function $c(\theta)$ on $\left[0,180^{\circ}\right)$, always greater than 1, such that if $\alpha \neq 0$ is not a root of unity all of whose conjugates lie in $S_{\theta}$, then $\Omega(\alpha) \geq c(\theta)$. Note that this result is not numerical. G. Rhin and C. J. Smyth [Rhin and Smyth 95] gave a lower bound for $c(\theta)$. Moreover, they succeeded in finding the exact value of $c(\theta)$ for $\theta$ in nine subintervals of $\left[0,120^{\circ}\right]$ and conjectured that $c(\theta)$ is a step function of $\theta$ that is constant except for finitely many left discontinuities in any closed subinterval of $\left[0,180^{\circ}\right)$. Their method uses explicit auxiliary polynomials $A(X)=X^{a} R(X)$, where $R$ is a reciprocal polynomial in $\mathbb{Z}[X]$, found by heuristic methods.
More recently, Q. Wu [Wu 03] developed an algorithm to obtain explicit upper bounds for the norm of polynomials related to the integer transfinite diameter and thus to find polynomials that must be involved in such auxiliary functions. Hence, instead of a heuristic search, he used a systematic search of suitable polynomials. His algorithm allowed him and G. Rhin [Rhin and Wu 04] to compute polynomials with minimal norm on $S(\theta) \cap\{z \in \mathbb{C}:|z| \leq 1\}$. They gave the exact value of $c(\theta)$ for four new intervals of $\left[0,140^{\circ}\right]$ and extended four existing intervals.

Throughout this paper, we will consider another height function, the trace, defined as follows: let $\alpha$ be an algebraic integer of degree $d \geq 1$, and $P$ its minimal polynomial:

$$
P=X^{d}+a_{1} X^{d-1}+\cdots+a_{d}=\prod_{i=1}^{d}\left(X-\alpha_{i}\right) .
$$

The trace of $\alpha$ (and of $P$ ) is $\operatorname{trace}(\alpha)=\sum_{i=1}^{d} \alpha_{i}=$ $-a_{1}$, and the absolute trace of $\alpha$ (and of $P$ ) is $\mathcal{T}(\alpha)=$ $\operatorname{trace}(\alpha) / d$.

Then any set $E_{\theta}$ with $0 \leq \theta<90^{\circ}$ equipped with the trace satisfies the Northcott property, and we will be interested in studying its small points.

First, we summarize the known results for the trace when $\theta=0$ (for a complete survey, see [Aguirre and Peral 08]). Then we explain how we have extended the study of $\mathcal{T}$ for $0<\theta<90^{\circ}$. The functions $\Omega$ and $\mathcal{T}$ are of different natures ( $\Omega$ is multiplicative and $\mathcal{T}$ is additive), but their behavior in their domains of definition is very similar: see [Rhin and Smyth 95] and Theorem 1.2 below.

\subsection{The Case $\theta=0$}

The case $\theta=0$ is known as the Schur-Siegel-Smyth trace problem.

Problem 1.1. Fix $\rho<2$. Then show that all but finitely many totally positive algebraic integers $\alpha$ have $\mathcal{T}(\alpha)>\rho$.

Following work of I. Schur [Schur 18] and C. L. Siegel [Siegel 45], C. J. Smyth [Smyth 84b] proved that $\mathcal{T}(\alpha)>$ 1.7719 with a finite set of exceptions. More recently, this bound was raised to 1.7783786 by J. F. McKee and C. J. Smyth [McKee and Smyth 04] in 2004, to 1.784109 by J. Aguirre and J. C. Peral [Aguirre and Peral 08] in 2006, and then to 1.78702 by the author [Flammang 08] in 2007. In contrast to the previous works, in which the authors used heuristic methods involving only totally positive polynomials, this last result was obtained 
with a more sophisticated version of Wu's algorithm and involved several polynomials with complex roots. The set of exceptions is composed of the roots of the polynomials $X, X-1, X^{2}-3 X+1, X^{3}-5 X^{2}+6 X-1$, $X^{4}-7 X^{3}+13 X^{2}-7 X+1$, and $X^{4}-7 X^{3}+14 X^{2}-8 X+1$. In this paper the usual integer transfinite diameter was replaced by a generalized integer transfinite diameter. This method was first used in [Flammang et al. 06].

The Case $\theta>0$. All these results lead us naturally to seek a lower bound for the absolute trace of algebraic integers all of whose conjugates lie in a sector $S_{\theta}$ with $0<\theta<90^{\circ}$.

We prove the following theorem.

Theorem 1.2. There exist a left discontinuous, strictly positive step function $g$ on $\left[0,90^{\circ}\right)$ and a positive, continuous, monotonically decreasing function $f$ on $\left[0,90^{\circ}\right)$ such that for $\theta \in\left[0,90^{\circ}\right)$, if $\alpha$ is a nonzero algebraic integer whose conjugates all lie in $S_{\theta}$, then

$$
\mathcal{T}(\alpha) \geq \min (f(\theta), g(\theta))
$$

unless the minimal polynomial of $\alpha$ is equal to one of $X$, $X-1, X^{2}-3 X+1, X^{3}-5 X^{2}+6 X-1$.

Moreover, the exact value of $v(\theta)=\inf _{|\arg \alpha| \leq \theta} \mathcal{T}(\alpha)$ is known on seven subintervals of $\left[0,90^{\circ}\right)$.

These intervals are given in Table 1.

Let $\left(P_{i}\right)_{1 \leq i \leq 21}$ be the polynomials given in Table 2 and $\theta_{i}=\min _{\theta>0}\left\{P_{i} \in S_{\theta}\right\}$. The function $g$ is defined by

$$
g(\theta)=\mathcal{T}\left(P_{i}\right)
$$

for $1 \leq i \leq 21, \theta_{i} \leq \theta<\theta_{i+1}$, where $\theta_{22}=90^{\circ}$.

The function $f(\theta)$ is given by $f(\theta)=\max _{1 \leq i \leq 7}\left(f_{i}(\theta)\right)$, and the functions $f_{i}(\theta)$ are defined by

$$
f_{i}(\theta)=\min _{z \in S_{\theta}}\left(\Re(z)-\sum_{1 \leq j \leq J} c_{i j} \log \left|Q_{i j}(z)\right|\right),
$$

where the polynomials $Q_{i j}$ and the real numbers $c_{i j}$ are read off from Table 3, using polynomials of Table 4.

Remark 1.3. We give a numerical example for the function $g$. If $21.64038404 \leq \theta \leq 24.07$, then $f_{3}(\theta)=$ $1.33333584>g(\theta)=\frac{4}{3}$, so that $v(\theta)=g(\theta)=\frac{4}{3}=$ $\mathcal{T}\left(P_{8}\right)$. For $\theta>24.07$, then $\frac{4}{3}=g(\theta)>f_{3}(\theta)$, so that $v(\theta) \geq f_{3}(\theta)$.

In Section 2, we explain the method of explicit auxiliary functions used to obtain inequality (1-1). In Section

\begin{tabular}{cccccc}
$i$ & $v(\theta)$ & $\theta_{i}$ & $\theta^{\prime}{ }_{i}$ & \multicolumn{2}{c}{$P$} \\
\hline 1 & 1.75000000 & 0.00000000 & 2.58 & $P_{1}$ & or $P_{1}^{\prime}$ \\
2 & 1.33333333 & 21.64038404 & 24.07 & $P_{8}$ & \\
3 & 1.25000000 & 26.40874008 & 27.9 & $P_{9}$ & \\
4 & 1.00000000 & 38.66828249 & 39.42 & $P_{11}$ \\
5 & 0.75000000 & 49.35368062 & 52.04 & $P_{13}$ \\
6 & 0.50000000 & 60.00000000 & 65.17 & $P_{16}$ \\
7 & 0.25000000 & 76.79562995 & 77.58 & $P_{19}$ \\
\hline
\end{tabular}

TABLE 1. Intervals $\left[\theta_{i}, \theta^{\prime}{ }_{i}\right]$ where $v(\theta)$ is known exactly. Here $v(\theta)=v\left(\theta_{i}\right)=\mathcal{T}(P)$ for $\theta \in\left[\theta_{i}, \theta^{\prime}{ }_{i}\right]$. The polynomials $P$ are read off from Table 2 . The angles are given in degrees.

3, we link explicit auxiliary functions to a generalization of the classical integer transfinite diameter. In Section 4, we explain how Wu's algorithm is used to produce the polynomials $Q_{i j}$ that appear in the functions $f_{i}$.

All the computations were done on an iBook Macintosh with the languages Pascal and Pari.

\section{THE EXPLICIT AUXILIARY FUNCTIONS}

The method of auxiliary functions was introduced by $\mathrm{C}$. J. Smyth [Smyth 81a, Smyth 81b] and is based on the fact that the resultant of two polynomials in $\mathbb{Z}[X]$ with no common roots is a nonzero integer. For simplicity, we explain the method of auxiliary functions in the case of the Schur-Siegel-Smyth trace problem.

Suppose that we are able to find a polynomial $Q \in$ $\mathbb{Z}[X]$ and real constants $a>0$ and $b$ such that

$$
x-a \log |Q(x)| \geq b \quad \text { for } x>0 .
$$

Then, if $\alpha$ is a totally positive algebraic integer with minimal polynomial $P$, we have

$$
\frac{1}{d} \operatorname{trace}(\alpha) \geq b+a \log |\operatorname{res}(P, Q)| .
$$

If we suppose that $Q(\alpha) \neq 0$, then $|\operatorname{res}(P, Q)| \geq 1$, and the inequality $(2-1)$ gives the lower bound $b$ for the absolute trace of $\alpha$.

C. J. Smyth [Smyth 84b] computed explicitly a polynomial $Q \in \mathbb{Z}[X]$ and a constant $a>0$ such that

$$
x-a \log |Q(x)| \geq 1.7719 \text { for } x>0 .
$$

The function $x-a \log |Q(x)|$ is called an explicit auxiliary function.

If we replace $x$ by $\log \max (1,|x|)$, we obtain a lower bound for the Weil height of an algebraic integer. For examples of applications of this method to other height functions, see [Rhin and $\mathrm{Wu} 06]$. 


\begin{tabular}{lccl} 
& $\mathcal{T}(P)$ & $\theta$ & \multicolumn{1}{c}{$P$} \\
\hline$P_{1}$ & 1.75000000 & 0.00000000 & $1-7 x+13 x^{2}-7 x^{3}+x^{4}$ \\
$P_{1}^{\prime}$ & 1.75000000 & 0.00000000 & $1-8 x+14 x^{2}-7 x^{3}+x^{4}$ \\
$P_{2}$ & 1.71428571 & 4.68737270 & $-1+15 x-69 x^{2}+133 x^{3}-121 x^{4}+55 x^{5}-12 x^{6}+x^{7}$ \\
$P_{3}$ & 1.66666666 & 10.91503368 & $-1+18 x-116 x^{2}+357 x^{3}-590 x^{4}+551 x^{5}-298 x^{6}+92 x^{7}-15 x^{8}+x^{9}$ \\
$P_{4}$ & 1.62500000 & 12.29344727 & $1-17 x+100 x^{2}-244 x^{3}+293 x^{4}-191 x^{5}+69 x^{6}-13 x^{7}+x^{8}$ \\
$P_{5}$ & 1.60000000 & 13.52174930 & $-1+10 x-25 x^{2}+22 x^{3}-8 x^{4}+x^{5}$ \\
$P_{6}$ & 1.57142857 & 14.12571478 & $-1+13 x-60 x^{2}+115 x^{3}-104 x^{4}+48 x^{5}-11 x^{6}+x^{7}$ \\
$P_{7}$ & 1.50000000 & 17.77114822 & $1-18 x+128 x^{2}-455 x^{3}+904 x^{4}-1065 x^{5}+774 x^{6}-351 x^{7}+97 x^{8}-15 x^{9}+x^{10}$ \\
$P_{8}$ & 1.33333333 & 21.64038404 & $-1+5 x-4 x^{2}+x^{3}$ \\
$P_{9}$ & 1.25000000 & 26.40874008 & $1-5 x+9 x^{2}-5 x^{3}+x^{4}$ \\
$P_{10}$ & 1.16666666 & 32.56993995 & $1-8 x+25 x^{2}-32 x^{3}+21 x^{4}-7 x^{5}+x^{6}$ \\
$P_{11}$ & 1.00000000 & 38.66828249 & $1-4 x+7 x^{2}-4 x^{3}+x^{4}$ \\
$P_{12}$ & 0.83333333 & 47.94143201 & $1-5 x+13 x^{2}-17 x^{3}+13 x^{4}-5 x^{5}+x^{6}$ \\
$P_{13}$ & 0.75000000 & 49.35368062 & $1-3 x+5 x^{2}-3 x^{3}+x^{4}$ \\
$P_{14}$ & 0.66666666 & 59.01576957 & $-1+3 x-2 x^{2}+x^{3}$ \\
$P_{15}$ & 0.62500000 & 59.36024037 & $1-5 x+16 x^{2}-29 x^{3}+37 x^{4}-29 x^{5}+16 x^{6}-5 x^{7}+x^{8}$ \\
$P_{16}$ & 0.50000000 & 60.00000000 & $1-x+x^{2}$ \\
$P_{17}$ & 0.37500000 & 73.00145189 & $1-3 x+10 x^{2}-14 x^{3}+20 x^{4}-14 x^{5}+10 x^{6}-3 x^{7}+x^{8}$ \\
$P_{18}$ & 0.33333333 & 76.25367928 & $1-3 x+7 x^{2}-6 x^{3}+6 x^{4}-2 x^{5}+x^{6}$ \\
$P_{19}$ & 0.25000000 & 76.79562995 & $1-x+3 x^{2}-x^{3}+x^{4}$ \\
$P_{20}$ & 0.16666666 & 84.84160375 & $1-x+5 x^{2}-3 x^{3}+5 x^{4}-x^{5}+x^{6}$ \\
$P_{21}$ & 0.12500000 & 85.94398603 & $1-x+7 x^{2}-4 x^{3}+13 x^{4}-4 x^{5}+7 x^{6}-x^{7}+x^{8}$ \\
\hline & & &
\end{tabular}

TABLE 2. Polynomials used in the function $g$ of Theorem 1.2.

\begin{tabular}{|c|c|c|c|}
\hline$i$ & $\theta_{i}^{\prime}$ & $Q_{i j}$ & $c_{i j}$ \\
\hline 1 & 2.58 & $\begin{array}{llllll}Q_{0} & Q_{1} & Q_{2} & Q_{3} & Q_{4} & Q_{5} \\
Q_{6} & Q_{7} & Q_{8} & Q_{10} & Q_{11} & Q_{12} \\
Q_{13} & Q_{14} & Q_{15} & Q_{16} & Q_{17}\end{array}$ & $\begin{array}{lllllll}0.56284363 & 0.50408065 & 0.08166128 & 0.20590509 & 0.09015004 & 0.02224684 \\
0.03236765 & 0.00649871 & 0.00145134 & 0.01170589 & 0.00369868 & 0.01800095 \\
0.00402967 & 0.00791983 & 0.00286599 & 0.00225963 & 0.01031189 & \end{array}$ \\
\hline 2 & 24.07 & $\begin{array}{llll}Q_{0} & Q_{1} & Q_{18} & Q_{19} \\
Q_{20} & Q_{21} & Q_{24}\end{array}$ & $\begin{array}{llll}0.54006460 & 0.68965168 & 0.06390586 & 0.00197963 \\
0.00491943 & 0.03327370 & 0.01811781\end{array}$ \\
\hline 3 & 27.9 & $\begin{array}{llll}Q_{0} & Q_{1} & Q_{18} & Q_{20} \\
Q_{21} & Q_{22} & Q_{23} & Q_{25}\end{array}$ & $\begin{array}{llll}0.50621857 & 0.67788105 & 0.05256367 & 0.01508746 \\
0.04076393 & 0.00857661 & 0.00568225 & 0.00367281\end{array}$ \\
\hline 4 & 39.42 & $\begin{array}{llll}Q_{0} & Q_{1} & Q_{26} & Q_{27} \\
Q_{28} & Q_{29} & Q_{32} & Q_{37}\end{array}$ & $\begin{array}{llll}0.44972962 & 0.60721238 & 0.01919192 & 0.00387718 \\
0.02639647 & 0.01309434 & 0.00189094 & 0.00334521\end{array}$ \\
\hline 5 & 52.04 & $\begin{array}{lllll}Q_{0} & Q_{30} & Q_{31} & Q_{33} & Q_{34} \\
Q_{35} & Q_{36} & Q_{37} & Q_{38}\end{array}$ & $\begin{array}{llllll}0.39607424 & 0.01895977 & 0.01051677 & 0.05454688 & 0.00445482 \\
0.00801740 & 0.00023269 & 0.00766288 & 0.10140545\end{array}$ \\
\hline 6 & 65.17 & $\begin{array}{llll}Q_{0} & Q_{38} & Q_{39} & Q_{40} \\
Q_{41} & Q_{42} & Q_{43} & \end{array}$ & $\begin{array}{llll}0.29210698 & 0.07386767 & 0.00160050 & 0.02498593 \\
0.00544326 & 0.00560379 & 0.00704141\end{array}$ \\
\hline 7 & 77.58 & $\begin{array}{llll}Q_{0} & Q_{44} & Q_{45} & Q_{46} \\
Q_{47} & Q_{48} & Q_{49} & Q_{50}\end{array}$ & $\begin{array}{llll}0.13924563 & 0.01356400 & 0.00064003 & 0.00895138 \\
0.00327017 & 0.00245762 & 0.00084550 & 0.00488970\end{array}$ \\
\hline
\end{tabular}

TABLE 3. The auxiliary functions $f_{i}, 1 \leq i \leq 7$. 


\begin{tabular}{|c|c|c|c|}
\hline & $\mathcal{T}(Q)$ & $\varphi(Q)$ & $Q$ \\
\hline$\overline{Q_{0}}$ & 0.00000000 & 0.00000000 & $x$ \\
\hline$Q_{1}$ & 1.00000000 & 0.00000000 & $-1+x$ \\
\hline$Q_{2}$ & 2.00000000 & 0.00000000 & $-2+x$ \\
\hline$Q_{3}$ & 1.50000000 & 0.00000000 & $1-3 x+x^{2}$ \\
\hline$Q_{4}$ & 1.66666666 & 0.00000000 & $-1+6 x-5 x^{2}+x^{3}$ \\
\hline$Q_{5}$ & 1.75000000 & 0.00000000 & $1-7 x+13 x^{2}-7 x^{3}+x^{4}$ \\
\hline$Q_{6}$ & 1.75000000 & 0.00000000 & $1-8 x+14 x^{2}-7 x^{3}+x^{4}$ \\
\hline$Q_{7}$ & 1.80000000 & 0.00000000 & $-1+12 x-31 x^{2}+27 x^{3}-9 x^{4}+x^{5}$ \\
\hline$Q_{8}$ & 1.80000000 & 0.00000000 & $-1+13 x-32 x^{2}+27 x^{3}-9 x^{4}+x^{5}$ \\
\hline$Q_{9}$ & 2.00000000 & 0.00000000 & $1-4 x+x^{2}$ \\
\hline$Q_{10}$ & 2.00000000 & 0.00000000 & $2-4 x+x^{2}$ \\
\hline$Q_{11}$ & 1.83333333 & 3.17650728 & $\begin{array}{l}1-29 x+318 x^{2}-1726 x^{3}+5233 x^{4}-9481 x^{5}+10709 x^{6}-7760 x^{7} \\
+3652 x^{8}-1107 x^{9}+208 x^{10}-22 x^{11}+x^{12}\end{array}$ \\
\hline$Q_{12}$ & 1.71428571 & 4.68737270 & $-1+15 x-69 x^{2}+133 x^{3}-121 x^{4}+55 x^{5}-12 x^{6}+x^{7}$ \\
\hline$Q_{13}$ & 1.75000000 & 5.69698026 & $\begin{array}{l}1-27 x+281 x^{2}-1470 x^{3}+4336 x^{4}-7742 x^{5}+8750 x^{6}-6430 x^{7} \\
+3102 x^{8}-972 x^{9}+190 x^{10}-21 x^{11}+x^{12}\end{array}$ \\
\hline$Q_{14}$ & 1.75000000 & 6.23618248 & $1-19 x+111 x^{2}-277 x^{3}+339 x^{4}-221 x^{5}+78 x^{6}-14 x^{7}+x^{8}$ \\
\hline$Q_{15}$ & 1.75000000 & 6.42782898 & $1-21 x+120 x^{2}-289 x^{3}+345 x^{4}-222 x^{5}+78 x^{6}-14 x^{7}+x^{8}$ \\
\hline$Q_{16}$ & 1.75000000 & 10.23941165 & $\begin{array}{l}1-27 x+283 x^{2}-1483 x^{3}+4372 x^{4}-7789 x^{5}+8780 x^{6}-6439 x^{7} \\
+3103 x^{8}-972 x^{9}+190 x^{10}-21 x^{11}+x^{12}\end{array}$ \\
\hline$Q_{17}$ & 1.60000000 & 13.52174930 & $-1+10 x-25 x^{2}+22 x^{3}-8 x^{4}+x^{5}$ \\
\hline$Q_{18}$ & 1.33333333 & 21.64038404 & $-1+5 x-4 x^{2}+x^{3}$ \\
\hline$Q_{19}$ & 1.37500000 & 25.19411073 & $1-13 x+62 x^{2}-146 x^{3}+181 x^{4}-127 x^{5}+51 x^{6}-11 x^{7}+x^{8}$ \\
\hline$Q_{20}$ & 1.40000000 & 25.53545872 & $-1+7 x-18 x^{2}+17 x^{3}-7 x^{4}+x^{5}$ \\
\hline$Q_{21}$ & 1.25000000 & 26.40874008 & $1-5 x+9 x^{2}-5 x^{3}+x^{4}$ \\
\hline$Q_{22}$ & 1.33333333 & 27.82114255 & $1-9 x+31 x^{2}-41 x^{3}+26 x^{4}-8 x^{5}+x^{6}$ \\
\hline$Q_{23}$ & 1.00000000 & 28.62953072 & $1-6 x+12 x^{2}-8 x^{3}+2 x^{4}$ \\
\hline$Q_{24}$ & 1.28571428 & 32.42286109 & $-1+10 x-39 x^{2}+72 x^{3}-67 x^{4}+34 x^{5}-9 x^{6}+x^{7}$ \\
\hline$Q_{25}$ & 1.16666666 & 32.56993995 & $1-8 x+25 x^{2}-32 x^{3}+21 x^{4}-7 x^{5}+x^{6}$ \\
\hline$Q_{26}$ & 1.00000000 & 38.66828249 & $1-4 x+7 x^{2}-4 x^{3}+x^{4}$ \\
\hline$Q_{27}$ & 0.93750000 & 40.46899673 & $1-9 x+38 x^{2}-88 x^{3}+122 x^{4}-102 x^{5}+52 x^{6}-15 x^{7}+2 x^{8}$ \\
\hline$Q_{28}$ & 1.00000000 & 40.89484445 & $-1+4 x-3 x^{2}+x^{3}$ \\
\hline$Q_{29}$ & 1.00000000 & 41.92031705 & $1-7 x+20 x^{2}-25 x^{3}+17 x^{4}-6 x^{5}+x^{6}$ \\
\hline$Q_{30}$ & 1.00000000 & 45.00000000 & $2-2 x+x^{2}$ \\
\hline$Q_{31}$ & 1.00000000 & 45.17331986 & $1-6 x+17 x^{2}-22 x^{3}+16 x^{4}-6 x^{5}+x^{6}$ \\
\hline$Q_{32}$ & 0.75000000 & 45.65507052 & $1-4 x+8 x^{2}-6 x^{3}+2 x^{4}$ \\
\hline$Q_{33}$ & 0.75000000 & 49.35368062 & $1-3 x+5 x^{2}-3 x^{3}+x^{4}$ \\
\hline$Q_{34}$ & 0.80000000 & 51.65190097 & $-1+5 x-9 x^{2}+9 x^{3}-4 x^{4}+x^{5}$ \\
\hline$Q_{35}$ & 0.83333333 & 52.16543234 & $1-5 x+14 x^{2}-18 x^{3}+13 x^{4}-5 x^{5}+x^{6}$ \\
\hline$Q_{36}$ & 0.68750000 & 52.92437629 & $1-7 x+26 x^{2}-53 x^{3}+70 x^{4}-59 x^{5}+33 x^{6}-11 x^{7}+2 x^{8}$ \\
\hline$Q_{37}$ & 0.75000000 & 53.35032016 & $1-6 x+20 x^{2}-38 x^{3}+48 x^{4}-38 x^{5}+20 x^{6}-6 x^{7}+x^{8}$ \\
\hline$Q_{38}$ & 0.50000000 & 60.00000000 & $1-x+x^{2}$ \\
\hline$Q_{39}$ & 0.50000000 & 62.67373071 & $1-5 x+17 x^{2}-31 x^{3}+42 x^{4}-35 x^{5}+22 x^{6}-8 x^{7}+2 x^{8}$ \\
\hline$Q_{40}$ & 0.50000000 & 64.08635381 & $1-2 x+4 x^{2}-2 x^{3}+x^{4}$ \\
\hline$Q_{41}$ & 0.50000000 & 66.90163222 & $1-3 x+9 x^{2}-9 x^{3}+8 x^{4}-3 x^{5}+x^{6}$ \\
\hline$Q_{42}$ & 0.50000000 & 67.87659748 & $1-4 x+13 x^{2}-22 x^{3}+29 x^{4}-22 x^{5}+13 x^{6}-4 x^{7}+x^{8}$ \\
\hline$Q_{43}$ & 0.50000000 & 69.29518894 & $2-x+x^{2}$ \\
\hline$Q_{44}$ & 0.25000000 & 76.79562995 & $1-x+3 x^{2}-x^{3}+x^{4}$ \\
\hline$Q_{45}$ & 0.33333333 & 77.57978776 & $1-2 x+6 x^{2}-5 x^{3}+6 x^{4}-2 x^{5}+x^{6}$ \\
\hline$Q_{46}$ & 0.25000000 & 78.65759095 & $1-2 x+8 x^{2}-9 x^{3}+15 x^{4}-9 x^{5}+8 x^{6}-2 x^{7}+x^{8}$ \\
\hline$Q_{47}$ & 0.33333333 & 81.60136144 & $-1+3 x-11 x^{2}+17 x^{3}-24 x^{4}+22 x^{5}-16 x^{6}+9 x^{7}-3 x^{8}+x^{9}$ \\
\hline$Q_{48}$ & 0.36363636 & 81.61660173 & $-1+3 x-15 x^{2}+28 x^{3}-52 x^{4}+60 x^{5}-62 x^{6}+46 x^{7}-28 x^{8}+13 x^{9}-4 x^{10}+x^{11}$ \\
\hline$Q_{49}$ & 0.30000000 & 86.19355737 & $1-2 x+11 x^{2}-16 x^{3}+32 x^{4}-30 x^{5}+32 x^{6}-18 x^{7}+11 x^{8}-3 x^{9}+x^{10}$ \\
\hline$Q_{50}$ & 0.00000000 & 90.00000000 & $1+x^{2}$ \\
\hline
\end{tabular}

TABLE 4. Polynomials used in the auxiliary functions $f_{i}$ where $\varphi(Q)=\max \{|\arg z|: Q(z)=0\}$.

In our case, the explicit auxiliary functions $f_{i}$ are of the following type $\left(\theta \in\left[0,90^{\circ}\right)\right.$ fixed): for $z \in S_{\theta}$,

$$
\begin{aligned}
f_{\theta}(z) & =\Re(z)-a \log |Q(x)| \\
& =\Re(z)-\sum_{1 \leq j \leq J} c_{j} \log \left|Q_{j}(z)\right| \geq m_{\theta},
\end{aligned}
$$

where the polynomials $Q_{j}$ are the irreducible factors of the polynomial $Q$. Therefore, we have $\mathcal{T}(\alpha) \geq m_{\theta}$, and we get a lower bound for the absolute trace of $\alpha$.

The main problem is then to find a good list of polynomials $Q_{j}$ that gives a value of $m_{\theta}$ as large as possible. Thus, we link the auxiliary function (2-2) with a gener- 
alization of the integer transfinite diameter in order to find our polynomials $Q_{j}$ by Wu's algorithm.

\section{AUXILIARY FUNCTIONS AND INTEGER TRANSFINITE DIAMETER}

In this section, we will need the following definition:

Definition 3.1. Let $K$ be a compact subset of $\mathbb{C}$. The integer $f$-transfinite diameter of $K$, where $f: K \rightarrow \mathbb{R}^{+}$ is an upper semicontinuous function, is defined by

$$
t_{\mathbb{Z}, f}(K)=\liminf _{\substack{n \geq 1 \\ n \rightarrow+\infty}} \inf _{\substack{P \in \mathbb{Z}[X] \\ \operatorname{deg}(P)=n}} \sup _{z \in K}\left(|P(z)|^{1 / n} f(z)\right) .
$$

This weighted version of the integer transfinite diameter was introduced by F. Amoroso [Amoroso 93] and is an important tool in the study of rational approximations of logarithms of rational numbers.

Let $\theta$ be a fixed angle in $\left[0,90^{\circ}\right)$. Here, we deal with the compact $K$ replaced by the unbounded subset $S_{\theta}$ of $\mathbb{C}$. So we choose a weight $\varphi$ such that $t_{\mathbb{Z}, \varphi}\left(S_{\theta}\right)$ is a finite number, which allows us to define the integer $\varphi$ transfinite diameter of the sector $S_{\theta}$.

Inside the auxiliary function (2-2) we replace the number $a$ by a rational number. So we may write that for $z \in S_{\theta}$

$$
f_{\theta}(z)=\Re(z)-\frac{t}{r} \log |Q(z)| \geq m_{\theta},
$$

where $Q \in \mathbb{Z}[X]$ is of degree $r$ and $t$ is a positive real number. We want to get a function $f_{\theta}$ whose minimum $m_{\theta}$ in the sector $S_{\theta}$ is as large as possible. Thus we search for a polynomial $Q \in \mathbb{Z}[X]$ such that

$$
\sup _{z \in S_{\theta}}|Q(z)|^{t / r} e^{-\Re(z)} \leq e^{-m_{\theta}} .
$$

If we suppose that $t$ is fixed, we need to get an effective upper bound for the quantity

$$
t_{\mathbb{Z}, \varphi}\left(S_{\theta}\right)=\liminf _{\substack{r \geq 1 \\ r \rightarrow+\infty}} \inf _{\substack{P \in \mathbb{Z}[X] \\ \operatorname{deg}(P)=r}} \sup _{z \in S_{\theta}}\left(|P(z)|^{t / r} \varphi(z)\right),
$$

where we use the weight $\varphi(z)=e^{-\Re(z)}$. It is clearly a generalization of the integer $f$-transfinite diameter of a compact set of $\mathbb{C}$.

\section{CONSTRUCTION OF THE AUXILIARY FUNCTIONS}

\subsection{How to Find the Polynomials $Q_{j}$}

Let $\theta$ be a fixed angle in $\left[0,90^{\circ}\right)$. The auxiliary function $f_{\theta}$ is harmonic outside a finite set containing the roots of the polynomials $Q_{j}$, and so it takes its minimum in the sector $S_{\theta}$ on the boundary of $S_{\theta}$. It is clear that $f_{\theta}(z) \rightarrow \infty$ as $z \rightarrow \infty$. So since $f_{\theta}(z)=f_{\theta}(\bar{z})$, it is sufficient to search for the minimum of $f$ on the upper edge of the sector $S_{\theta}$ where $z=x e^{i \theta}$ with $x>0$.

We take an initial value of $t$, say $t_{0}=1$, and a finite set $F_{0}$ of control points on the edge of $S_{\theta}$. After several numerical attempts, it appears that the set

$$
\begin{gathered}
F_{0}=\left\{x e^{i \theta}: x \text { belongs to a set of } 50\right. \text { points } \\
\text { uniformly distributed on }[0,2.5]\}
\end{gathered}
$$

gives good results.

The idea is to use Wu's algorithm [Wu 03] to compute a polynomial $Q(z)=\sum_{k=0}^{d} a_{k} z^{k}$ in $\mathbb{Z}[z]$ that is small on $F_{0}$, and then take its irreducible factors as polynomials $Q_{j}$. We usually take $d=15$.

In order to use LLL in this algorithm, for any $x e^{i \theta}$ in $F_{0}$, we replace the quantity $\sum_{k=0}^{d} a_{k} x e^{i \theta}$, which is not a real linear form in the unknown coefficients $a_{k}$, by its real and imaginary parts. Therefore, we search for a polynomial $Q$ such that these two quantities are small on $F_{0}$. We define $Q_{1}$ as the irreducible factor of $Q$ of smallest degree, and we take the best value of $c_{1}$ to get the best auxiliary function $f_{\theta, 1}$. We deduce from this the value $t_{1}=c_{1} \operatorname{deg}\left(Q_{1}\right)$.

We add to the set $F_{0}$ the points for which $f_{\theta, 1}$ has a local minimum (including those where $x$ is greater than $2.5)$ to get a new set $F_{1}$ of control points. With Wu's algorithm we compute a polynomial $Q$ of degree $d+\operatorname{deg} Q_{1}$ that is a multiple of $Q_{1}$ of small norm on $F_{1}$ and take $Q_{2}$ as another irreducible factor of $Q$. We optimize $\left(c_{1}, c_{2}\right)$ to get the best function $f_{\theta, 2}$. This gives $t_{2}$.

We get the set $F_{2}$ from $F_{1}$ by adding the local minima of $f_{\theta, 2}$. Then we search for a polynomial $Q$ that is a multiple of $Q_{1} Q_{2}$ of degree $d+\operatorname{deg} Q_{1}+\operatorname{deg} Q_{2}$, and we continue this process until two consecutive steps produce no new polynomial.

\subsection{Optimization of the $c_{j}$}

Let $\theta$ be a fixed angle in $\left[0,90^{\circ}\right)$. For the optimization of the auxiliary function $f_{\theta}$ we use the semi-infinite linear programming method introduced into number theory by Smyth [Smyth 84b]. We recall it briefly. We define by induction a sequence of finite sets $X_{n}, n \geq 0$, with $X_{n} \subset S_{\theta}$. We start with an arbitrary set of points $X_{0}$ of cardinality greater than $J$. At each step $n \geq 0$, we compute the best values for $c_{j}$ by linear programming on the set $X_{n}$.

We get a function $f_{\theta, n}$ whose minimum $m_{\theta, n}=$ $\min _{x \in X_{n}} f_{\theta, n}(x)$ is greater than $m_{\theta, n}^{\prime}=\min _{x \in S_{\theta}} f_{\theta, n}(x)$. 
We add to $X_{n}$ the points of $S_{\theta}$ for which $f_{\theta, n}$ has a local minimum smaller than $m_{\theta, n}+\epsilon_{n}$, where $\left(\epsilon_{n}\right)_{n \geq 0}$ is a decreasing sequence of positive numbers tending to 0 when $n$ is increasing and chosen such that the set $X_{n}$ does not increase too quickly. We stop, for instance, when $m_{\theta, n}-m_{\theta, n}^{\prime}<10^{-6}$. If $k$ steps are necessary, we take $m_{\theta}=m_{\theta, k}^{\prime}$.

\section{CONCLUSION}

It seems reasonable to think that the method described above, which allows us to find "small points" for several sets $E_{\theta}$ equipped with the trace, can be applied to other sets of algebraic numbers that satisfy the Northcott property.

\section{ACKNOWLEDGMENTS}

The author wishes to thank Professor Georges Rhin for his valuable help in this work.

\section{REFERENCES}

[Aguirre and Peral 08] J. Aguirre and J.C. Peral. "The Trace Problem for Totally Positive Algebraic Integers." In Number Theory and Polynomials, edited by J. F. McKee and C. J. Smyth, London Math. Society Lecture Note Series 352. Cambridge, UK: Cambridge University Press, 2008.

[Amoroso 93] F. Amoroso. " $f$-Transfinite Diameter and Theoretic Applications." Ann. Inst. Fourier, Grenoble 43 (1993), 1179-1198.

[Amoroso and Dvornicich 00] F. Amoroso and R. Dvornicich. "A Lower Bound for the Height in Abelian Extensions." J. Number Theory 80:2 (2000), 260-272.

[Bombieri 08] E. Bombieri. "Problems and Results on the Distribution of Algebraic Points on Algebraic Varieties." To appear in J. Théor. Nombres Bordeaux, 2008.

[Borwein and Erdélyi 96] P. Borwein and T. Erdélyi. "The Integer Chebyshev Problem. Math. Comp. 65 (1996), 661681.

[Flammang 96] V. Flammang. "Two New Points in the Spectrum of the Absolute Mahler Measure of Totally Positive Algebraic Integers." Math. Comp. 65 (1996), 307-311.

[Flammang 08] V. Flammang. "Trace of Totally Positive Algebraic Integers and Integer Transfinite Diameter." To appear in Math. Comp., 2008.

[Flammang et al. 97] V. Flammang, G. Rhin, and C. J. Smyth. "The Integer Transfinite Diameter of Intervals and Totally Real Algebraic Integers." J. Théor. Nombres Bordeaux 9 (1997), 137-168.
[Flammang et al. 06] V. Flammang, G. Rhin, and J. M. SacEpée. "Integer Transfinite Diameter and Polynomials with Small Mahler Measure." Math. Comp. 255 (2006), 15271540 .

[Langevin 86] M. Langevin. "Minorations de la maison et de la mesure de Mahler de certains entiers algébriques." $C$. $R$. Acad. Sci. Paris 303 (1986), 523-526.

[Lehmer 33] D. H. Lehmer. "Factorization of Certain Cyclotomic Functions." Ann. of Math. (2) 34:3 (1933), 461-479.

[McKee and Smyth 04] J. F. McKee and C. J. Smyth. "Salem Numbers of Trace -2 and Trace of Totally Positive Algebraic Integers." ANTS 2004, LNCS 3076 (2004), 327-337.

[Rhin and Smyth 95] G. Rhin and C. J. Smyth. "On the Absolute Mahler Measure of Polynomials Having All Zeros in a Sector." Math. Comp. 64 (1995), 295-304.

[Rhin and $\mathrm{Wu}$ 04] G. Rhin and C. Wu. "On the Absolute Mahler Measure of Polynomials Having All Zeros in a Sector, II." Math. Comp. 74 (2004), 383-388.

[Rhin and Wu 06] G. Rhin and C. Wu. "Integer Transfinite Diameter and Computation of Polynomials." LMAM 200614 (2006). Available at http://www.math.univ-metz.fr/ $\sim$ rhin/prepint_bristol.pdf

[Schinzel 73] A. Schinzel. "On the Product of the Conjugates outside the Unit Circle." Acta Arith. 24 (1973), 385-399.

[Schur 18] I. Schur. "Über die Verteilung der Wurzeln bei gewissen algebraischen Gleichungen mit ganzzahligen Koeffizienten." Math. Z. 1 (1918), 377-402.

[Siegel 45] C. L. Siegel. "The Trace of Totally Positive and Real Algebraic Integers." Ann. of Math. 46 (1945), 302312 .

[Smyth 71] C. J. Smyth. "On the Product of the Conjugates outside the Unit Circle of an Algebraic Integer." Bull. London Math. Soc. 3 (1971), 169-175.

[Smyth 81a] C. J. Smyth. "On the Measure of Totally Real Algebraic Integers." J. Austral. Math. Soc. 30 (1980-1981), 137-149.

[Smyth 81b] C. J. Smyth. "On the Measure of Totally Real Algebraic Integers II." J. Austral. Math. Soc. 37 (1981), 205-208.

[Smyth 84a] C. J. Smyth. "The Mean Value of Totally Real Algebraic Numbers." Math. Comp. 42 (1984), 663-681.

[Smyth 84b] C. J. Smyth. "Totally Positive Algebraic Integers of Small Trace." Ann. Inst. Fourrier, Grenoble 33 (1984), 1-28.

[Smyth 08] C. J. Smyth. "The Mahler Measure of Algebraic Numbers: A Survey." In Number Theory and Polynomials, edited by J. F. McKee and C. J. Smyth, London Math. Society Lecture Note Series 352. Cambridge, UK: Cambridge University Press, 2008.

[Wu 03] Q. Wu. "On the Linear Independence Measure of Logarithms of Rational Numbers." Math. Comp. 72 (2003), 901-911. 
V. Flammang, UMR CNRS 7122 Département de Mathématiques, UFR MIM, Université Paul Verlaine de Metz, Ile du Saulcy, 57045 Metz cedex 01, France (flammang@univ-metz.fr)

Received November 12, 2007; accepted in revised form April 29, 2008. 